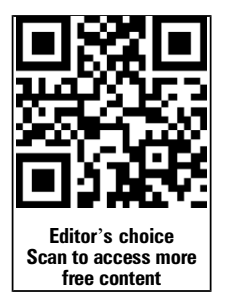

Center for Clinical

Epidemiology, Odense University Hospital and Research Unit of Clinical Epidemiology, Institute of Clinical Research, University of Southern Denmark, Odense, Denmark

${ }^{2}$ Crohn's and Colitis Center, Brigham and Women's Hospital, Boston, Massachusetts, USA ${ }^{3}$ Harvard Medical School, Harvard University, Boston, Massachusetts, USA ${ }^{4}$ Department $\mathrm{D}$, Centre of Andrology and Fertility Clinic, Odense University Hospital, Odense, Denmark

${ }^{5}$ Research Unit of Human Reproduction, Institute of Clinical Research, University of Southern Denmark, Odense, Denmark

\section{Correspondence to} Professor Bente Mertz Nørgård, Center for Clinical Epidemiology, Odense University Hospital. Sdr. Boulevard 29, entrance 216, Odense C DK-5000, Denmark; bente.noergaard@rsyd.dk

Received 7 December 2015 Revised 18 January 2016 Accepted 8 February 2016 Published Online First 26 February 2016

\title{
Live birth and adverse birth outcomes in women with ulcerative colitis and Crohn's disease receiving assisted reproduction: a 20-year nationwide cohort study
}

\author{
B M Nørgård, ${ }^{1,2,3}$ P V Larsen, ${ }^{1}$ J Fedder, ${ }^{4,5}$ P S de Silva, ${ }^{2,3}$ M D Larsen, ${ }^{1}$ \\ S Friedman ${ }^{1,2,3}$
}

\begin{abstract}
Objective To examine the chance of live births and adverse birth outcomes in women with ulcerative colitis (UC) and Crohn's disease (CD) compared with women without inflammatory bowel disease (IBD) who have undergone assisted reproductive technology (ART) treatments.
\end{abstract}

Methods This was a nationwide cohort study based on Danish health registries, comprising all women with an embryo transfer during 1 January 1994 through 2013. The cohorts comprised 1360 ART treatments in 432 women with UC, 554 ART treatments in 182 women with CD and 148540 treatments in 52489 women without IBD. Our primary outcome was live births per ART treatment cycle. We controlled for multiple covariates in the analyses. Our secondary outcomes were adverse birth outcomes.

Results The chance of a live birth for each embryo transfer was significantly reduced in ART treatments in women with UC (OR=0.73, $95 \% \mathrm{Cl} 0.58$ to 0.92 ), but not significantly reduced in the full model of ART treatments in women with $C D(O R=0.77,95 \% \mathrm{Cl} 0.52$ to 1.14). Surgery for CD before ART treatment significantly reduced the chance of live birth for each embryo transfer (OR=0.51, $95 \% \mathrm{Cl} 0.29$ to 0.91$)$. In children conceived through ART treatment by women with UC, the OR of preterm birth was $5.29(95 \% \mathrm{Cl}$ 2.41 to 11.63 ) in analyses including singletons and multiple births; restricted to singletons the OR was 1.80 , $95 \% \mathrm{Cl} 0.49$ to 6.62 .

Conclusions Our results suggest that women with UC and $C D$ receiving ART treatments cannot expect the same success for each embryo transfer as other infertile women. Women with CD may seek to initiate ART treatment before needing $C D$ surgery. Increased prenatal observation in UC pregnancies after ART should be considered.

\section{INTRODUCTION}

The incidence of ulcerative colitis (UC) and Crohn's disease (CD) continue to increase in many CrossMark

To cite: Nørgård BM, Larsen PV, Fedder J, et al. Gut 2016;65:767-776. countries and Denmark is one of the countries in the world with the highest reported incidence rate (23 per 100000 per year for UC and 10 per 100000 per year for CD). ${ }^{1-5}$ As the majority of patients with UC and CD are diagnosed in their fertile years, ${ }^{5}$ clinicians are increasingly faced with

\section{Significance of this study}

What is already known on this subject?

- Women with ulcerative colitis (UC) and Crohn's disease (CD) might have decreased fertility due to several factors, including voluntary childlessness, prior surgery, decreased ovarian reserve and increased disease activity.

- Assisted reproductive technology (ART) is an appropriate intervention for women with UC and $C D$ who are unable to conceive naturally.

- There is little evidence of the efficacy of ART treatment in women with UC and CD. The cumulative live birth rate after six cycles of in vitro fertilisation has only been described in 71 women with UC and 49 women with CD.

- No studies have examined the risk of adverse birth outcomes in children after ART treatments in women with $\mathrm{UC}$ and $\mathrm{CD}$.

\section{What are the new findings?}

- In women with UC receiving ART, the chance of a live birth for each embryo transfer is significantly reduced.

- UC surgery before ART treatment does not have a negative effect on the chance of live birth for each embryo transfer.

- In children conceived through ART treatment by women with UC, the risk of preterm birth is increased.

- In women with $C D$ receiving ART, the chance of a live birth for each embryo transfer is decreased (but not significant in the full model).

- CD surgery before ART treatment significantly reduces the chance of a live birth for each embryo transfer.

- In children conceived through ART treatment by women with $C D$, there is no increased risk of adverse birth outcomes.

issues relating to fertility and pregnancy. Pregnancy attempts in women with UC and CD are most often well planned and patients are often worried about the effects of medical or surgical treatment. 
Significance of this study

\section{How might it impact on clinical practice in the foreseeable future?}

- Infertile women with UC and CD may seek to initiate ART treatment more quickly if they cannot conceive naturally as they cannot expect the same success for each embryo transfer as other infertile women.

- Women with UC will be reassured that surgery before ART treatment does not affect the chance of a live birth for each embryo transfer.

- Increased prenatal observation in UC pregnancies after ART should be considered.

- Women with CD may seek to initiate ART treatment before needing $C D$ surgery.

- Women with CD will be reassured that children born after ART treatment have normal birth outcomes.

Some of the most urgent questions asked by patients of childbearing age concern fertility, safety of a future pregnancy, and risk of adverse birth outcomes.

In the general population in Denmark, up to $10-15 \%$ of all women have problems of infertility, leading many to seek assisted reproduction, ${ }^{6}{ }^{7}$ which includes in vitro fertilisation (IVF), with or without fertilisation with intracytoplasmic sperm injection (ICSI) and transfer of frozen-thawed (FER) embryos. The proportion of women with UC and CD and infertility problems is likely to be at least the same as in the general population or even higher owing to adhesions of the fallopian tubes and ovaries associated with prior abdominal surgery. ${ }^{8}$ Former studies have found a considerable reduction in postoperative fertility in women with UC requiring ileoanal pouch anastomosis (IPAA), ${ }^{9-12}$ and after CD surgery. ${ }^{13}{ }^{14}$ Excluding voluntary childlessness, additional causes of infertility may include increased disease activity and low ovarian reserve. ${ }^{13} 1516$ Serum anti-Mullerian hormone, an indicator of ovarian reserve, is lower in women with $\mathrm{CD}$, especially during periods of active disease, compared with healthy controls. ${ }^{15} 16$

Assisted reproductive technology (ART) is an appropriate fertility intervention for women with UC and $\mathrm{CD}$ who are unable to conceive naturally. Therefore, questions about the success of ART treatment in women with UC and CD are highly relevant, especially before surgery for these conditions. There is little evidence of the efficacy of ART treatment in patients with UC and CD. The cumulative live birth rate after six IVF cycles has only recently been described in 71 women with UC and $49 \mathrm{CD}$ women and compared with patients without IBD, ${ }^{17}{ }^{18}$ but the analyses did not take into account the possible effect of confounding factors which might affect fertility, such as prior surgery, comorbid disease, body mass index (BMI) and tobacco use.

We examined the efficacy of ART in women with UC and $\mathrm{CD}$, compared with women without IBD, on a much larger scale. In this study we used the power of the Danish nationwide registries, which contain all relevant information on ART treatments and births, and compiled data corresponding to a study period of 20 years on unselected patient cohorts. In Denmark, the cumulative proportion of infertility is $16-26 \%$ in women who try conceiving for $>12$ months and more than 15000 ART treatments are initiated each year. ${ }^{7}$ For this study, ART refers to traditional IVF, ICSI and FER. The primary outcome studied was live birth and we assessed the chance of a live birth after ART treatments in women with UC and those with CD compared with women without IBD. In subanalyses, we examined the impact of UC and CD surgery before ART treatment. As secondary outcomes, we compared the risk of adverse birth outcomes in children of women with UC and CD and women without IBD after ART treatment.

\section{MATERIALS AND METHODS}

\section{Study population and setting}

All citizens in Denmark (population approximately 5.6 million inhabitants, $>90 \%$ Caucasians) have equal and free access to the tax-supported healthcare system, and its uniform organisation allowed us to use a nationwide population-based design. We obtained nationwide Danish data from the following registries: (i) data related to ART treatments and cause of infertility from the Danish ART Registry, (ii) data on the primary outcome of infertility treatment (live birth) from the Danish Medical Birth Registry (MBR), (iii) data from the Danish National Patient Registry (NPR) on comorbid diseases and surgical procedures performed in women with UC and CD and (iv) data from the Central Personal Registration system on death and immigration. Data from all sources were unambiguously linked on an individual level using the unique civil registration number (assigned to all Danish residents at birth from the Central Personal Registration system) and used in all Danish healthcare registries. ${ }^{19}$

The study population comprised all women (with a valid civil registration number and available for follow-up in Denmark) registered in the ART registry with at least one embryo transfer during the study period of 1 January 1994 to 31 December 2013 (20-year study period). The Danish healthcare system offers infertile couples and single women up to three fully reimbursed IVF or ICSI treatment cycles with fresh embryos and an unlimited number of frozen embryo transfer and insemination cycles (in practice, 3-6 cycles) if the woman is aged $<40$ years. All treatment cycles for infertility are recorded in the Danish ART registry. The registry was established on 1 January 1994 and includes data on ART treatments in both public and private clinics; registration of all treatments is compulsory. ${ }^{20} 21$ In the ART registry, the embryo transfer to the uterus might be a result of all kinds of preceding treatments (IVF, ICSI, FER).

\section{Exposed cohorts}

From the study population, all women with UC and CD were identified in the NPR. The NPR includes records of all patients discharged from Danish hospitals since 1977 and all outpatient visits since 1994. ${ }^{22}{ }^{23}$ Information in the NPR includes patients' civil registration numbers, hospital, department, dates of admission and discharge, procedures performed and up to 20 discharge diagnoses based on the International Classification of Diseases (ICD-8 before 1994 and ICD-10 from 1994 onward). All eligible patients who had a discharge history of UC from any hospital in Denmark (ICD-8 codes: 563.19, 569.04; ICD-10 codes: DK51.x (except DK51.9 for unspecified disease)) before the date of embryo transfer were identified. If a woman with UC had a former diagnosis in the NPR with codes of CD she was only included if the latest given diagnosis was UC. Similarly, all eligible patients who had a discharge history of CD (ICD-8 codes: 563.01; ICD-10 codes: DK50.x) before the date of embryo transfer were identified. If a woman with a CD diagnosis had a former diagnosis of UC she was only included if the latest given diagnosis was $\mathrm{CD}$. 
For the primary outcome (live birth), the exposed cohorts comprised all ART treatment cycles in women with UC or CD. Because each woman with UC or CD could have several ART treatments, the observation unit was the treatment cycle. ${ }^{24-26}$

For the secondary outcomes (adverse birth outcomes), the exposed cohorts comprised all children who were conceived through ART treatment by women with UC or CD. Because each woman with UC or CD could have several births and each birth could include several children, the observation unit was the child.

\section{Unexposed cohorts}

For the primary outcome, the unexposed cohort comprised all ART treatment cycles in women without UC or CD (women without IBD), and the observation unit was the treatment cycle.

For the secondary outcomes, the unexposed cohort comprised all children born after ART treatment by women without IBD, and the observation unit was the child.

\section{Primary and secondary outcomes}

The primary outcome was live birth within a period of 124292 days after the date of each embryo transfer and was identified in the MBR. Thus, a live birth was considered to be the result of the particular ART treatment if the difference was 140-308 days (20-44 weeks) ${ }^{24}$ from the last menstruation start, corresponding to 124-292 days after embryo transfer. The MBR includes information on all births in Denmark since 1 January 1973 and data are obtained from birth forms, completed by the midwives who attend all births. ${ }^{27} 28$ The forms include data on the mother's civil registration number, date of birth, parity and smoking status during pregnancy, as well as gestational age, birth weight and congenital anomalies (CAs).

Our secondary outcomes included (i) low birth weight (LBW) at term (birth weight $<2500 \mathrm{~g}$ in children with a gestational age of $\geq 37$ weeks), (ii) preterm birth (birth before 37 completed weeks of pregnancy) and (iii) CAs.

\section{Data on confounders}

Covariates were selected a priori. From the NPR we obtained data on comorbid diseases for all women in the study population and a comorbidity index score-the Charlson Index score, was calculated for each treatment cycle of each woman and covers 19 major disease categories weighted according to their prognostic impact. ${ }^{29}$ We computed the index based on diagnoses recorded during all previous admissions to hospital since 1977, and two index levels were defined: no comorbidity (Charlson Index 0), some comorbidity (Charlson Index $\geq 1$ ). From the MBR we obtained data on parity $(0,+1)$ and smoking during pregnancy (yes/no). The ART registry did not include information on all confounders during the entire study period but for that period we had data on women's age at time of embryo transfer (continuous variable), calendar year of infertility treatment (1994-1998, 1999-2003, 2004-2008, 20092013), type of preceding treatment (IVF, ICSI, FER) and cause of infertility (female factor, male factor, or mixture of factors/ idiopathic). From 2006, the ART registry also included information on BMI (calculated as body weight divided by height squared and categorised according to the WHO classifications as underweight $\left(<18.5 \mathrm{~kg} / \mathrm{m}^{2}\right)$, normal weight $\left(18.5-24.9 \mathrm{~kg} / \mathrm{m}^{2}\right)$, overweight $\left(25.0-29.9 \mathrm{~kg} / \mathrm{m}^{2}\right)$ and obese $\left.\left(\geq 30 \mathrm{~kg} / \mathrm{m}^{2}\right)\right)$, partner's age, alcohol intake (yes/no) and smoking at the time of embryo transfer (yes/no).

For women with UC we calculated the duration of UC at the time of each ART treatment (calculated from the date that the first diagnosis of UC appeared in the NPR, unless they had a later diagnosis of $\mathrm{CD}$, until the date of embryo transfer in the ART registry). Likewise, the disease duration for $\mathrm{CD}$ women was calculated from the date that the first diagnosis of $\mathrm{CD}$ appeared in the NPR, unless they had a later diagnosis of UC, until the date of embryo transfer.

For women with UC and CD we identified those who had undergone surgery before the date of embryo transfer. Identified were women with UC who had relevant operations according to the following codes in the new and former version of the "Nordic Classification of Surgical Procedures": codes of KJFH; that is, all types of colectomies; and colectomy codes of 45020 , 45060 , 45080, 45840, or 45880. For CD women the following codes were used: KJFB00, KJFB01, KJFB20/21/30/31, KJFB33, KJFB34，KJFB40/41/43/44/46/47/50/51/60/61/63/64, KJFB96, KJFB97， KJFH00/01/10/11/20/96， KJGB00/01/10/11/30/31, KJFA60, 43440, 43460, 43520, 43540, 43680, 43700, 43740, 43760, 43780, 43800, 43820, 43840, 43860, 43880, 44060, $44120,44150,44160,44790,44900,44920,44940,44960$, 44980, 45020, 45060, 45080, 45100, 45120, 45200, 45240, $45320,45480,45690,45840,45860,45880,46290$. The number of UC- or CD-related surgeries was recorded before each treatment cycle.

\section{Statistical analyses}

Live birth (primary outcome)

Contingency tables were constructed for the main study variables according to the exposed and unexposed cohorts. We used multilevel logistic regression analyses to compute crude and adjusted relative risk estimates (prevalence OR with 95\% CIs) for live births following ART treatments in women with UC and $\mathrm{CD}$ relative to women without IBD and the model accounted for multiple embryo transfers in the same woman. Adjustment was made for Charlson Index, women's age at the time of embryo transfer, calendar year of infertility treatment, type of infertility treatment and cause of infertility. In an extended model, including data from 2006, we further adjusted for BMI, partner's age, smoking at the time of embryo transfer and alcohol.

In a sensitivity analysis we included only first time ART treatment for each woman with UC or CD and only if they had no missing information on BMI, smoking at the time of embryo transfer and alcohol.

\section{Subanalysis on the impact of UC or CD surgery on the chance of live birth}

A possible impact of UC or CD surgery before infertility treatment was examined in women with UC or CD, respectively. Thus, for women with UC who had received ART treatments, two subcohorts were established: (a) an exposed cohort who had had at least one UC-related surgery before the date of embryo transfer and (b) an unexposed cohort who had not had surgery before the date of embryo transfer. Live birth in the exposed cohort (a) was compared with that in the unexposed cohort (b). Similarly, for women with CD who had received ART treatments, exposed and unexposed cohorts were established. In multilevel logistic regression models we adjusted for the duration of UC or CD, Charlson Index, women's age, calendar year of infertility treatment, type of infertility treatment and cause of infertility. Owing to lack of power it was not possible to adjust for smoking at the time of embryo transfer, BMI and alcohol.

All surgery codes given to women with UC at the three latest operation dates before ART treatment were recorded according to the following categories: (i) 'partial colon resection (no 
ostomy)': 45020, (ii) 'total colon resection (no ostomy)': 45060, KJFH00, KJFH96, (iii) 'total colon resection+ileostomy (rectum left in place)': KJFH10, KJFH11, (iv) 'rectal/anus resection': 45080, 45840, 45880, KJFH20, (v) 'ileal pouch anal anastomosis': KJFH33. All CD surgery codes from the three latest operation dates before the first embryo transfer were identified and the surgery codes were categorised according to (i) 'ileocecal resection': 43700, KJFB20, KJFB21, (ii) 'ileocolonic resection (more than ileocecal)': 45060, 43860, 43820, 44900, 43800, 43880, 43840, KJFB33, KJFB30, KJFH00, (iii) 'small bowel resection or stricturoplasty': 43680, 44060, 44790, 43780, 43760, 43440, 44120, KJFB00, KJFA60, (iv) 'stomas but rectum left in place': 43740, 45240, KJFH10, KJFB60, (v) 'colon resection': 44980, 44940, 45120, 44960, 44920, 45320, 45020, KJFB43, KJFB40, KJFB46, (vi) 'rectal/anus resection': 45880, 45840, 45080, 45860, KJGB30, KJFH20.

\section{Adverse birth outcomes (secondary outcomes)}

We used multilevel logistic regression analyses, accounting for multiple children by the same woman, to compute crude and adjusted relative risk estimates for adverse birth outcomes (LBW at term, preterm birth and CAs) after ART treatment in children of women with UC compared with children of women without IBD; a similar comparison was made for the children of women with CD. The risk estimates of LBW at term, preterm birth and CAs were adjusted for mother's age, parity, calendar year of infertility treatment, type of infertility treatment, cause of infertility and smoking during pregnancy. Estimates of LBW at term and CAs were further adjusted for singleton/multiple births.

In a subanalysis we used multilevel logistic regression to compare the risk of adverse birth outcomes after ART treatment among singletons of women with UC with singletons of women without IBD; a similar comparison was made for singletons of women with CD.

All analyses were conducted using Stata V.13 software (StataCorp LP, College Station, Texas, USA).

\section{RESULTS}

Table 1 shows the characteristics for all ART treatments in exposed and unexposed cohorts. A total of 1360 ART treatments in 432 women with UC and 554 ART treatments in 182 women with CD were included during the study period of 1 January 1994 to 31 December 2013. The unexposed cohort included 148540 ART treatments in 52489 women without IBD. The median age of women and the distribution according to BMI and smoking were similar in the exposed and unexposed cohorts, whereas fewer women with CD consumed alcohol than women with UC and unexposed women. In the UC cohort, the cause of infertility was referred to as a female factor in $34.5 \%$ of cases and a mixture of factors in $44.5 \%$ of cases; the corresponding figures for the CD cohort were $46.6 \%$ and $36.9 \%$ and for the unexposed cohort $30.1 \%$ and $38.0 \%$, respectively. The vast majority of women in all cohorts had no comorbid diseases. Among embryo transfers in patients with UC, the median duration of UC at the time of embryo transfer was 8 years $(25-75$ centiles: 4-12) and surgery had been performed before the transfer in $34.5 \%$ of cases. For patients with CD, the median duration of $\mathrm{CD}$ at the time of embryo transfer was 9 years (2575 centiles: $4-13$ ) and surgery had been performed before the transfer in $64.1 \%$ of cases.

\section{Live born children (primary outcome)}

The ORs for ART treatment in women with UC or CD leading to a live birth, compared with women without IBD, are given in table 2. The chance of a live birth was significantly reduced for each ART treatment in women with UC, with a crude $\mathrm{OR}=0.79$ (95\% CI 0.67 to 0.92 ). Two different models of adjustment were used but the ORs were virtually unchanged from the crude OR (in the fully adjusted model, $\mathrm{OR}=0.73$, $95 \%$ CI 0.58 to 0.92 ). The chance of a live birth was significantly reduced for each ART treatment in women with CD according to the crude OR $(0.62,95 \%$ CI 0.48 to 0.80$)$. In the fully adjusted model the chance of a live birth was still reduced but not significantly $(\mathrm{OR}=0.77,95 \% \mathrm{CI} 0.52$ to 1.14$)$. In the model containing no lifestyle factors, the OR was significantly reduced (table 2 ).

In the sensitivity analyses, we included only first-time ART treatment for each woman with UC and CD and only if she had non-missing information on lifestyle factors (BMI, smoking at the time of embryo transfer, alcohol). For women with UC, the result of this analysis was similar to the overall result of live birth (adjusted OR $=0.79,95 \%$ CI 0.53 to 1.18 ), and for CD also, the result was similar to the overall result, with a decreased, but not significant, chance of live birth.

\section{Subanalysis of the impact of UC or CD surgery on the chance of live birth}

Overall results for a possible impact of UC or CD surgery before infertility treatment on the chance of a live birth are given in table 3 . For UC, the results suggested no negative impact of former surgery on the chance of live birth. The result was very robust across the crude and adjusted OR (crude $\mathrm{OR}=0.92,95 \% \mathrm{CI} 0.64$ to 1.31 and adjusted $\mathrm{OR}=0.91,95 \% \mathrm{CI}$ 0.61 to 1.36 ). We examined all types of UC-relevant surgical procedures on the three latest dates of operations for all UC patients before their first ART cycle. We found that 195 procedures were performed and with a distribution as follows (the most common procedure first): (a) total colon resection (no ostomy), $\mathrm{N}=60$ $(30.8 \%)$; (b) total colon resection+ileostomy (rectum left in place), $\mathrm{N}=53$ (27.2\%); (c) rectal/anus resection, $\mathrm{N}=45$ (23.1\%); (d) ileal pouch anal anastomosis, $\mathrm{N}=36(18.5 \%)$ and (e) partial colon resection (no ostomy), $\mathrm{N}=1(0.51 \%)$.

For $\mathrm{CD}$, the results suggested that former surgery had a negative impact on the chance of live birth (crude OR $=0.56 ; 95 \%$ CI 0.33 to 0.93 and adjusted $O R=0.51 ; 95 \%$ CI 0.29 to 0.91 ). For women with $\mathrm{CD}$, the three latest operation dates before the first embryo transfer were identified; 206 procedures were performed with a distribution as follows: (a) ileocecal resection', $\mathrm{N}=50$ (24.3\%); (b) 'ileocolonic resection (more than ileocecal)', $\mathrm{N}=44 \quad(21.4 \%) ; \quad$ (c) 'small bowel resection or stricturoplasty', $\mathrm{N}=42$ (20.4\%); (d) 'stomas but rectum left in place', $\mathrm{N}=26$ (12.6\%); (e) 'colon resection', $\mathrm{N}=23$ (11.2\%) 'and (f) rectal/anus resection', $\mathrm{N}=21(10.2 \%)$.

\section{Adverse birth outcomes (secondary outcomes)}

Details of adverse birth outcomes are given in table 4 for singletons and multiple births. Children of women with UC had a significantly increased risk of preterm birth in comparison with children of women without IBD $(\mathrm{OR}=5.29$, 95\% CI 2.41 to 11.63). The relative risks of $\mathrm{LBW}$ at term and CAs were $\mathrm{OR}=1.48$ (95\% CI 0.69 to 3.13$)$ and $\mathrm{OR}=1.52$ (95\% CI 0.87 to 2.64), respectively. Children of women with $\mathrm{CD}$ had no significantly increased risk of preterm birth, LBW at term, or CAs in comparison with children of women without IBD (preterm birth: adjusted $\mathrm{OR}=0.44 ; 95 \% \mathrm{CI} 0.09$ to 2.21 ; LBW at term: adjusted $\mathrm{OR}=1.41$; $95 \%$ CI 0.40 to 4.89 ; CAs: adjusted $\mathrm{OR}=0.59 ; 95 \%$ CI 0.19 to 1.79 ). 
Table 1 Descriptive characteristics of study cohorts of assisted reproductive technology (ART) treatments in women with UC, CD and without IBD during the study period of 1 January 1994 through 2013

\begin{tabular}{|c|c|c|c|}
\hline Characteristics & $\begin{array}{l}\text { Exposed cohort (embryo transfers } \\
\text { in women with UC) } \\
(\mathrm{N}=1360)^{*}+\end{array}$ & $\begin{array}{l}\text { Exposed cohort (embryo transfers } \\
\text { in women with } \mathrm{CD}) \\
(\mathrm{N}=554)^{\star} \ddagger\end{array}$ & $\begin{array}{l}\text { Unexposed cohort (embryo transfers } \\
\text { in women without IBD) } \\
(\mathrm{N}=148540)^{\star} \S\end{array}$ \\
\hline \multicolumn{4}{|l|}{ Age at embryo transfer } \\
\hline Median ( $25-75$ centiles) & $33(30-36)$ & $32(30-35)$ & $33(30-37)$ \\
\hline \multicolumn{4}{|l|}{ Partner's age at embryo transfer } \\
\hline Median ( $25-75$ centiles) & $35.5(32-39)$ & $35(31-37)$ & $35(32-39)$ \\
\hline \multicolumn{4}{|l|}{ Female/male factor } \\
\hline Female factor, N (\%) & $442(34.5)$ & $234(46.6)$ & $41011(30.1)$ \\
\hline Male factor, N (\%) & $269(21.0)$ & $83(16.5)$ & $43610(32.0)$ \\
\hline Mixture of factors/idiopathic N (\%) & $570(44.5)$ & $185(36.9)$ & $51852(38.0)$ \\
\hline \multicolumn{4}{|l|}{ Type of preceding treatment } \\
\hline IVF, N (\%) & $718(53.0)$ & $290(52.6)$ & $72971(49.3)$ \\
\hline ICSI, N (\%) & $403(29.7)$ & $166(30.1)$ & $51237(34.6)$ \\
\hline FER, N (\%) & $234(17.3)$ & $95(17.2)$ & $23713(16.0)$ \\
\hline \multicolumn{4}{|l|}{ BMI } \\
\hline$<18.5$ (underweight), N (\%) & $15(2.3)$ & $16(7.2)$ & $1765(3.1)$ \\
\hline $18.5-24.99$ (normal), N (\%) & $450(69.7)$ & $125(56.3)$ & $37082(64.4)$ \\
\hline 25.00-29.99 (overweight), N (\%) & $134(20.7)$ & $69(31.1)$ & $13157(22.9)$ \\
\hline$\geq 30.00$ (obese), $\mathrm{N}(\%)$ & $47(7.3)$ & $12(5.4)$ & $5548(9.6)$ \\
\hline \multicolumn{4}{|l|}{ Smoking at the time of embryo transfer } \\
\hline Non-smoker, N (\%) & $617(93.8)$ & $192(87.3)$ & $51819(90.0)$ \\
\hline Smoker, N (\%) & $41(6.2)$ & $28(12.7)$ & $5773(10.0)$ \\
\hline \multicolumn{4}{|l|}{ Alcohol } \\
\hline No, $N(\%)$ & $304(52.0)$ & $135(70.3)$ & $29367(54.5)$ \\
\hline Yes, N (\%) & $281(48.0)$ & $57(29.7)$ & $24548(45.5)$ \\
\hline \multicolumn{4}{|l|}{ Calendar year of infertility treatment } \\
\hline $1994-1998, \mathrm{~N}(\%)$ & $110(8.1)$ & $84(15.2)$ & $24016(16.2)$ \\
\hline $1999-2003, \mathrm{~N}(\%)$ & $254(18.7)$ & $128(23.1)$ & $35899(24.2)$ \\
\hline $2004-2008, N(\%)$ & $469(34.5)$ & $188(33.9)$ & $44467(29.9)$ \\
\hline $2009-2013, N(\%)$ & $527(38.8)$ & $154(27.8)$ & $44158(29.7)$ \\
\hline \multicolumn{4}{|l|}{ Parity } \\
\hline $0, N(\%)$ & $169(62.4)$ & $65(70.7)$ & $24102(69.0)$ \\
\hline $1+, N(\%)$ & $102(37.6)$ & $27(29.3)$ & $10845(31.0)$ \\
\hline \multicolumn{4}{|l|}{ Comorbidity at embryo transfer } \\
\hline No comorbidity, N (\%) & $1202(88.4)$ & $476(85.9)$ & $136982(92.2)$ \\
\hline Some comorbidity, N (\%) & $158(11.6)$ & $78(14.1)$ & $11558(7.8)$ \\
\hline \multicolumn{4}{|c|}{ Duration of UC/CD at time of embryo transfer } \\
\hline Median (25-75 centiles) & $8(4-12)$ & $9(4-13)$ & - \\
\hline \multicolumn{4}{|c|}{ UC/CD operations before time of embryo transferf } \\
\hline No, $N(\%)$ & $891(65.5)$ & $199(35.9)$ & - \\
\hline Yes, $N(\%)$ & $469(34.5)$ & $355(64.1)$ & - \\
\hline \multicolumn{4}{|c|}{ Number of UC operations before embryo transferq } \\
\hline $1, N(\%)$ & $242(51.6)$ & - & - \\
\hline $2-3, N(\%)$ & $227(48.4)$ & - & - \\
\hline \multicolumn{4}{|c|}{ Number of CD operations before embryo transferq } \\
\hline $1, N(\%)$ & - & $175(49.3)$ & - \\
\hline $2-3, N(\%)$ & - & $159(44.8)$ & - \\
\hline$>3, N(\%)$ & - & $21(5.9)$ & - \\
\hline
\end{tabular}

*Number of women in exposed UC cohort=432, in exposed CD cohort=182 and in unexposed cohort=52 489.

tMissing (\%), women with UC: partner's age (42.5), female/male factor (5.8), type of treatment (0.4), parity (80.1), BMI (52.5), smoking at the time of embryo transfer (51.6), alcohol (57.0).

¥Missing (\%), women with CD: age of partner (49.6), fertility factor (9.4), ART treatment (0.5), parity (83.4), BMI (59.9), smoking at the time of embryo transfer (60.3), alcohol (65.3). §Missing (\%), women with no IBD: partner's age (53.4), female/male factor (8.1), type of treatment (0.4), parity (76.5), BMI (61.3), smoking at the time of embryo transfer (61.2), alcohol (63.7).

१II is possible to have more than one type of UC or CD operation on the same day.

BMI, body mass index; CD, Crohn's disease; FER, frozen-thawed embryos; IBD, inflammatory bowel disease; ICSI, intracytoplasmic sperm injection; IVF, in vitro fertilisation; UC, ulcerative colitis.

In a subanalysis we estimated the risk of adverse birth outcomes among singletons only and the results are given in table 5. Among singletons delivered by women with UC, the relative risks for LBW at term and CAs were close to unity and the relative risk of preterm birth was $\mathrm{OR}=1.80$ (95\% CI 0.49 to 6.62 ). For children delivered by women with $\mathrm{CD}$, we 
Table 2 Crude and adjusted ORs, with 95\% Cl, for live birth in the study cohorts of assisted reproductive technology (ART) treatments in women with UC compared with women without IBD; similar results for women with CD (1 January 1994 through 2013)

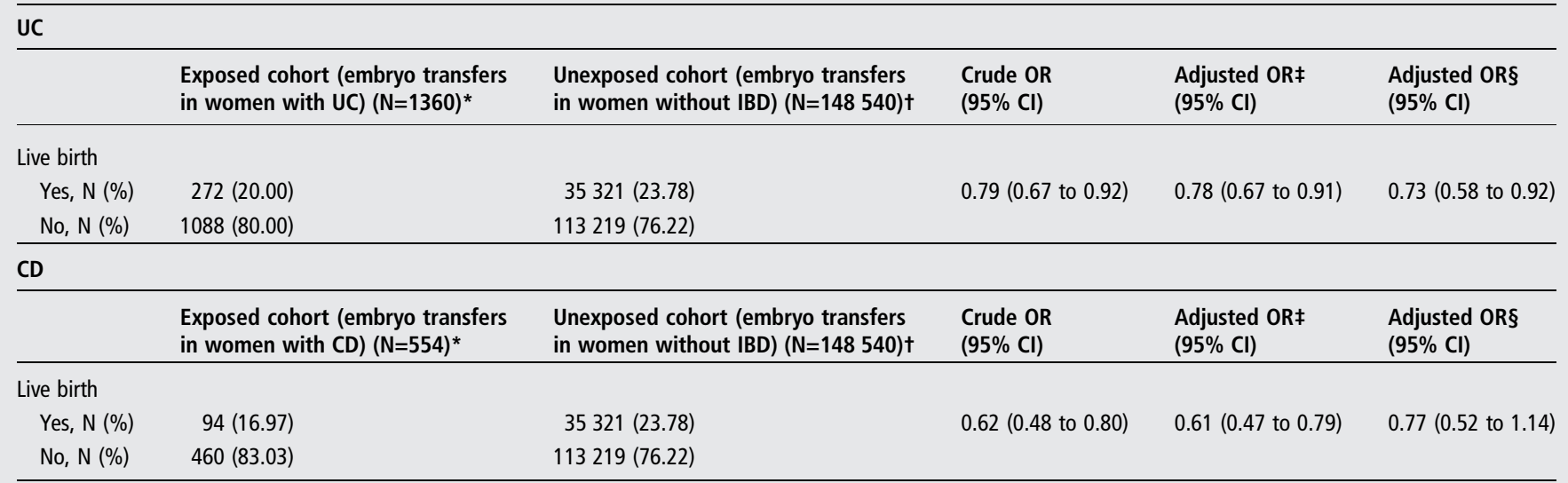

*Number of women in exposed UC cohort=432 and in exposed CD cohort=182. Intraclass correlation coefficient (ICC)=0.13.

tNumber of women in unexposed cohort $=52489$.

¥Adjusted for Charlson Index, women's age, calendar year of treatment, type of treatment (IVF, ICSI, FER), cause of infertility (female factor, male factor, or mixture of factors/ idiopathic). Number of observations $=137640$, number of women $=52015$.

§Extended model using data from 2006, adjusted for Charlson Index, women's age, calendar year of treatment, type of treatment (IVF, ICSI, FER), cause of infertility (female factor, male factor, or mixture of factors/idiopathic), BMI, partner's age, smoking at the time of embryo transfer and alcohol. Number of observations=48 575 , number of women=20 082 .

BMI, body mass index; CD, Crohn's disease; FER, frozen-thawed embryos; IBD, inflammatory bowel disease; ICSI, intracytoplasmic sperm injection; IVF, in vitro fertilisation; UC, ulcerative colitis.

found no significantly increased risk of preterm birth, LBW at term or CAs.

\section{DISCUSSION}

In this nationwide study with data from a span of 20 years, we found that ART treatments in women with UC and CD led to a reduced chance of a live birth per cycle, compared with ART treatments in women without IBD. Comparison of embryo transfers in women with CD who had had surgery before infertility treatment with those without prior surgery, showed a decreased chance of live births in women who had had surgery. After ART, the risk of preterm birth for children of women with UC was increased fivefold compared with children of women without IBD, but the risk disappeared when singletons only were evaluated.
This is the first study, based on nationwide data, analysing the chance of live birth after ART treatment in women with UC and CD. Earlier, the outcome of ART treatment was studied in 71 women with UC and 49 with CD from two tertiary care centres, ${ }^{17} 18$ who were compared with 470 controls from the general infertile population. It was found that the proportion of live births after the first ART cycle was similar among controls (30.2\%) and patients with UC (33.8\%) and CD (30.6\%). Our overall results differ from these findings. We found that among 1360 embryo transfers in women with UC and 554 embryo transfers in women with CD, there was a reduced chance of a live birth per cycle, compared with 148540 embryo transfers in women without IBD. It is worth noticing that this result is robust as the crude OR and the ORs from two adjusted models in both UC and CD were close. It might be argued that our

Table 3 Crude and adjusted ORs, with $95 \% \mathrm{Cl}$, for live birth in the cohort of assisted reproductive technology (ART) treatments in women with UC who had undergone surgery before infertility treatment, compared with ART treatments in women with UC who had not undergone surgery before infertility treatment; similar results for women with CD (1 January 1994 through 2013)

\begin{tabular}{|c|c|c|c|c|}
\hline \multicolumn{5}{|l|}{ UC } \\
\hline & $\begin{array}{l}\text { Exposed cohort (embryo transfers in women } \\
\text { with UC who had prior surgery) }(\mathrm{N}=469)^{*}\end{array}$ & $\begin{array}{l}\text { Unexposed cohort (embryo transfers in women } \\
\text { with UC who had no prior surgery) }(\mathrm{N}=891)^{*}\end{array}$ & $\begin{array}{l}\text { Crude OR } \\
(95 \% \mathrm{Cl})\end{array}$ & $\begin{array}{l}\text { Adjusted ORt } \\
(95 \% \mathrm{CI})\end{array}$ \\
\hline \multicolumn{5}{|l|}{ Live birth } \\
\hline Yes, N (\%) & 89 (18.98) & $183(20.54)$ & 0.92 (0.64 to 1.31$)$ & 0.91 (0.61 to 1.36$)$ \\
\hline No, N (\%) & $380(81.02)$ & 708 (79.46) & & \\
\hline \multicolumn{5}{|l|}{$C D$} \\
\hline & $\begin{array}{l}\text { Exposed cohort (embryo transfers in women } \\
\text { with } C D \text { who had prior surgery) }(\mathrm{N}=355) \ddagger\end{array}$ & $\begin{array}{l}\text { Unexposed cohort (embryo transfers in women } \\
\text { with CD who had no prior surgery) }(\mathrm{N}=199) \ddagger\end{array}$ & $\begin{array}{l}\text { Crude OR } \\
(95 \% \mathrm{Cl})\end{array}$ & $\begin{array}{l}\text { Adjusted ORT } \\
(95 \% \mathrm{Cl})\end{array}$ \\
\hline \multicolumn{5}{|l|}{ Live birth } \\
\hline Yes, N (\%) & $50(14.08)$ & $44(22.11)$ & 0.56 (0.33 to 0.93$)$ & $0.51(0.29$ to 0.91$)$ \\
\hline No, N (\%) & $305(85.92)$ & 155 (77.89) & & \\
\hline
\end{tabular}


Table 4 Crude and adjusted ORs, with $95 \% \mathrm{Cl}$, for adverse birth outcomes in the study cohorts of children after assisted reproductive technology (ART) in women with UC compared with children after ART in women without IBD; similar results in women with CD (1 January 1994 through 2013)

\begin{tabular}{|c|c|c|c|c|}
\hline \multicolumn{5}{|l|}{ UC } \\
\hline & $\begin{array}{l}\text { Exposed cohort* (embryo transfers } \\
\text { in women with UC) }\end{array}$ & $\begin{array}{l}\text { Unexposed cohort† (embryo transfers } \\
\text { in women without IBD) }\end{array}$ & $\begin{array}{l}\text { Crude OR } \\
(95 \% \mathrm{Cl})\end{array}$ & $\begin{array}{l}\text { Adjusted OR‡ } \\
(95 \% \mathrm{Cl})\end{array}$ \\
\hline \multicolumn{5}{|l|}{ Preterm birth§ } \\
\hline Yes, N (\%) & $100(30.77)$ & $9708(23.00)$ & 3.53 (1.72 to 7.22$)$ & $5.29(2.41$ to 11.63$)$ \\
\hline No, N (\%) & $225(69.23)$ & $32493(77.00)$ & & \\
\hline \multicolumn{5}{|l|}{ LBW at term } \\
\hline Yes, N (\%) & $13(5.75)$ & $1802(5.55)$ & 1.07 (0.49 to 2.30$)$ & $1.48(0.69 \text { to } 3.13)^{* *}$ \\
\hline No, N (\%) & $213(94.25)$ & $30684(94.45)$ & & \\
\hline \multicolumn{5}{|c|}{ Congenital abnormalities } \\
\hline Yes, N (\%) & $61(18.83)$ & $6652(15.73)$ & $1.29(0.77$ to 2.17$)$ & $1.52(0.87 \text { to } 2.64)^{* *}$ \\
\hline No, N (\%) & $263(81.17)$ & $35643(84.27)$ & & \\
\hline \multicolumn{5}{|l|}{$C D$} \\
\hline & $\begin{array}{l}\text { Exposed cohorttt (embryo transfers } \\
\text { in women with } C D \text { ) }\end{array}$ & $\begin{array}{l}\text { Unexposed cohortt(embryo transfers } \\
\text { in women without IBD) }\end{array}$ & $\begin{array}{l}\text { Crude OR } \\
(95 \% \mathrm{Cl})\end{array}$ & $\begin{array}{l}\text { Adjusted OR‡ } \\
(95 \% \mathrm{CI})\end{array}$ \\
\hline \multicolumn{5}{|l|}{ Preterm birth§ } \\
\hline Yes, N (\%) & $18(16.98)$ & $9708(23.00)$ & $0.42(0.09$ to 1.93$)$ & $0.44(0.09$ to 2.21$)$ \\
\hline No, N (\%) & $88(83.02)$ & $32493(77.00)$ & & \\
\hline \multicolumn{5}{|l|}{ LBW at term } \\
\hline Yes, N (\%) & $5(5.62)$ & $1802(5.55)$ & 0.99 (0.28 to 3.49$)$ & $1.41(0.40 \text { to } 4.89)^{* *}$ \\
\hline No, N (\%) & 84 (94.38) & $30684(94.45)$ & & \\
\hline \multicolumn{5}{|c|}{ Congenital abnormalities } \\
\hline Yes, N (\%) & $13(12.15)$ & $6652(15.73)$ & $0.70(0.27$ to 1.84$)$ & $0.59(0.19 \text { to } 1.79)^{* *}$ \\
\hline No, $N(\%)$ & $94(87.85)$ & $35643(84.27)$ & & \\
\hline \multicolumn{5}{|c|}{ 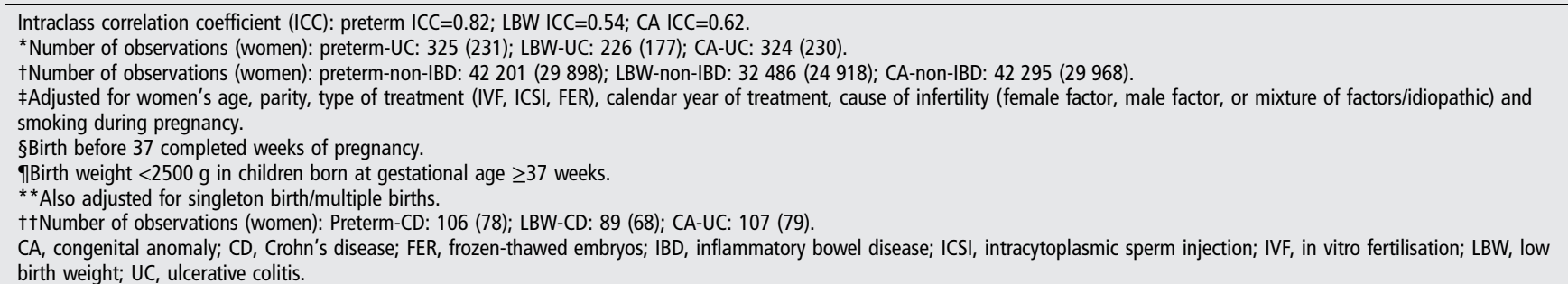 } \\
\hline
\end{tabular}

finding of a decreased chance of a live birth per cycle in women with UC and CD might not be related to the IBD itself but rather affected by IBD-related factors such as disease activity and/or medications used to treat IBD. The results in our study do not indicate which of these is the cause. It is unlikely that disease activity, at least at the stages of fertilisation and implantation, is the factor responsible for our results as women with IBD in Denmark are not referred for ART treatment unless they have quiescent disease. However, since this is a large registerbased study and it was not possible to review individual charts, we cannot rule out the possibility of a negative effect of active IBD, especially during a pregnancy. In addition, we were unable to examine medication use in our patients with IBD. Even if we had obtained information on IBD medication, we could not have adjusted for this in our main analyses as similar medication was not used in the unexposed cohort. Furthermore, as far as we can determine, no studies have indicated that underlying maternal medical treatment affects the result of ART treatments. It is reassuring that in several studies adalimumab, given before embryo transfer, improves the success of ART in women with immune-mediated infertility. ${ }^{30-32}$

Some results are emerging on the rates of adverse conception and pregnancy outcomes in general, without the use of ART, in women with IBD. Mahadevan's study of the Kaiser database in northern California ${ }^{33}$ and another recent meta-analysis ${ }^{34}$ indicated that women with IBD have adverse conception and pregnancy outcomes as well as an increased risk of pregnancy complications. The outcome of live birth can be affected at the stages of fertilisation, implantation and maintenance of the pregnancy throughout each trimester, but there is little knowledge of the factors that reduce the chance of a live birth or the most vulnerable stage (or stages) after ART treatment in women with IBD. Possibly, women with UC and CD have subclinical inflammation or increased immune factors not yet identified, ${ }^{35}$ such as antiphospholipid, antinuclear, antithyroid and antisperm antibodies or other undiscovered autoantibodies that decrease fertility; these underlying maternal factors may affect the chance of a live birth after ART.

In addition, initial research is now being done on microbiome changes during pregnancy in women without IBD. One study including 91 pregnant women showed significant changes in the gut microbiota during pregnancy. ${ }^{36}$ Specifically, Koren et al ${ }^{37}$ observed an overall increase in Proteobacteria and Actinobacteria from the first to third trimester, a decrease in Faecalibacteria and a decrease in the diversity of the microbiota. During pregnancy, a woman's immune system must adapt to avoid rejection of the fetus. At this time, the relationship between the observed changes in the microbiome and the 
Table 5 Crude and adjusted ORs, with $95 \% \mathrm{Cl}$, for adverse birth outcomes in singletons after assisted reproductive technology (ART) in women with UC compared with singletons after ART in women without IBD; similar results in women with CD (1 January 1994 through 2013)

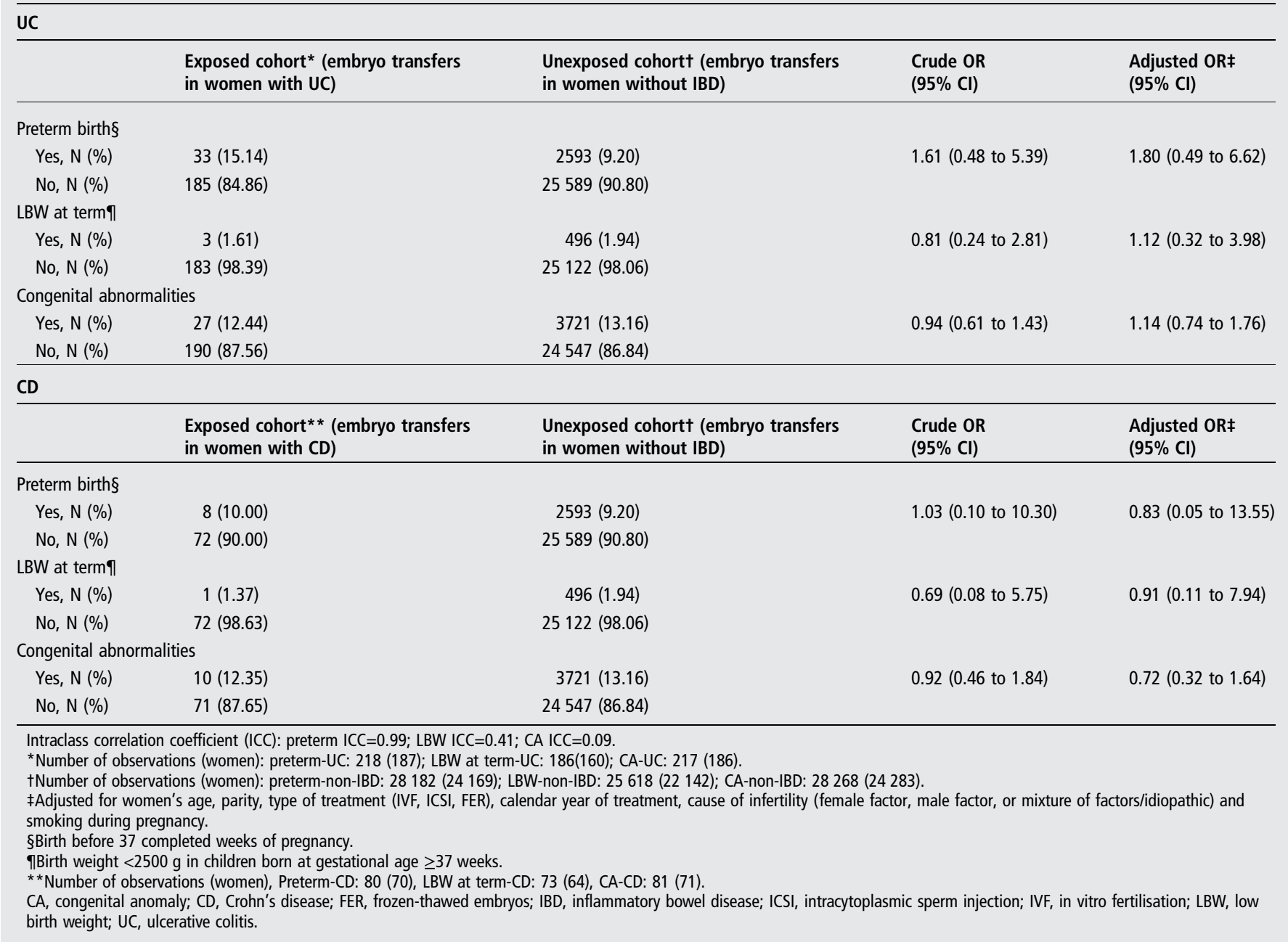

immune system and the role of the bacteria are not known. It has been hypothesised that the changes in microbiome may promote weight gain or may stimulate the innate immune response; the latter may affect the disease course during pregnancy. In addition, the impairment of innate immunity that has previously been reported in IBD may improve during pregnancy with changes in the microbiome and hormones that affect this immune response. The mechanism by which disease inflammation may be increased or decreased during pregnancy is not entirely understood. ${ }^{38} 39$ In pregnancy, the T helper 2 cell (Th2) responses are increased. CD is typically characterised by an exaggerated CD4T helper 1 (Th1) cell response. The increased Th2 response in pregnancy may affect the Th1 response that is upregulated in $\mathrm{CD}$, resulting in a decreased risk of flare. Since $\mathrm{UC}$ is a Th2-driven disease and Th2 responses are increased during pregnancy, patients with UC may have an increased risk of a flare. ${ }^{40}$

For the impact of surgery, it was suggested in the study by Pabby et al,,${ }^{17}$ based on the same 71 patients with UC as in the study by Oza et al, ${ }^{18}$ that the cumulative live birth rate after six ART cycles was comparable in women with UC with $(22$ patients), and without, IPAA (49 patients), but the study was descriptive and did not take confounders into consideration. In our study, 469 embryo transfers in women with UC and prior surgery were compared with 891 embryo transfers in women with UC without prior surgery and both the crude and the adjusted OR were close to unity, indicating that former surgery for UC did not have a negative impact on the chance of a live birth per cycle. The effect of surgery for CD on the chance of live birth after ART treatment has not been studied previously. In our study, 355 embryo transfers in women with CD and prior surgery were compared with 199 transfers in women with CD without prior surgery and both the crude and adjusted OR showed a significant decrease in live births after CD surgery. There are several possible explanations for these results. First, women with UC might have an increased risk of a flare during pregnancy owing to the increased Th2 response. An IPAA or ileostomy is a type of 'cure' which removes all present inflammation that might affect pregnancy. Therefore surgery in women with UC might not have a negative effect on live birth, especially since ART bypasses pelvic adhesions. Conversely, surgery for CD often does not remove all inflammation and CD commonly recurs after surgery. Possibly, also, patients with CD who need surgery have increased disease severity, which would account for the worse outcomes after ART treatment. These results are important when counselling women with UC and $\mathrm{CD}$ about surgery, if they are also considering a pregnancy.

Our study is the first to examine the risk of adverse birth outcomes in children after ART treatment in women with UC and $\mathrm{CD}$ and therefore our results cannot be compared with others. 
Our results suggest a significant fivefold increased risk of preterm birth in children after ART treatment in women with UC when the OR was adjusted for important confounders such as women's age, parity, type of ART treatment, calendar year of treatment, cause of infertility and smoking during pregnancy. Analysis of the risk of preterm birth in children among only singletons showed no significant increase. We did not find a significantly increased risk of LBW at term or CAs. Women with CD did not have an increased risk of adverse birth outcomes after ART treatment.

The precision and validity of our results depend on the size of the study, accurate classification of exposure, quality of outcome data and the ability to take into account the influence of confounders. According to these aspects our study has several strengths.

First, the study is based on a nationwide study population of all Danish women with UC and CD who had received ART treatment (and all other ART-treated women as controls) as we had access to the ART registry, which is based on mandatory reporting of all individual treatment cycles.

Second, the nature of the health registries allowed a 20-year study period and a large cohort of ART treatments, providing us with high statistical power.

Third, the data from the healthcare registries have a high degree of completeness and validity-that is, according to exposure assessment (diagnoses of $\mathrm{UC}$ and $\mathrm{CD}$ ). The completeness of diagnoses of UC and CD has been examined in a Danish study using the pathology system as a reference standard. This showed that of all patients with a confirmed diagnosis of UC or CD, 94\% were included in the NPR, which we had access to, and included obligatory registration of UC and CD diagnoses from both hospitals and outpatient visits. ${ }^{41}$ Furthermore, the overall validity of diagnosis of UC in the NPR was $94 \%$ and of CD 97\% in patients diagnosed at specialised departments. ${ }^{41}$ For our outcome measurements (live birth and adverse birth outcomes), the data from the MBR have a high degree of completeness and validity as all newborn children are registered in the $\mathrm{MBR}^{28}$

Fourth, we had information on several confounders, including the Charlson comorbidity Index, women's age, calendar year of treatment, type of infertility treatment, cause of infertility, BMI, partner's age, smoking at the time of embryo transfer, smoking during pregnancy and alcohol. Data on the type of operations performed in the patients with UC and CD are of high quality, as data from the NPR on surgical procedures have positive predictive values of $94-100 \%$.

Finally, our outcome data were obtained independently of the hypotheses investigated and exposure measurement, preventing differential misclassification of the outcome measurements.

The study also has some limitations. We lacked detailed information on the severity of UC and CD and the specific location of UC and CD lesions, although such information would only be relevant for the analysis comparing subcohorts of women with UC or CD. As discussed above, we had no information on disease activity and medical treatment for underlying maternal diseases in our cohorts. A further limitation is that we were unable to assess adverse birth outcomes among stillborn or very early abortions (including those due to antenatal diagnoses of CAs). Thus we might have underestimated the 'true' relative risks, if CAs after ART treatments, ending in stillbirth or very early abortions, are more prevalent in women with UC and CD than in women without IBD.

We conclude that women with $\mathrm{UC}$ and $\mathrm{CD}$ have a reduced chance of a live birth per ART treatment cycle compared with women without IBD. In addition, women with $\mathrm{CD}$, but not women with UC, with prior surgery have a decreased chance of a live birth per cycle. These findings might be related to the disease itself or to factors closely related to $\mathrm{UC}$ and $\mathrm{CD}$, and additional studies are needed in this area. Our results further suggest that increased prenatal observation in UC pregnancies after infertility treatment should be recommended. It is hoped that these results will inform treatment decisions about the use of ART in UC and CD and, particularly, recommendations for $\mathrm{CD}$ surgery and timing of attempts at conception. Future research will hopefully elucidate the mechanism of UC, CD and CD surgery on conception and maintenance of pregnancy. Also, to help with counselling, it would be interesting to examine the "take home chance" of having a baby per patient where the analytical unit is the woman and not the cycle.

Contributors BMN: funding, conception, design, data collection, assistance with data analysis, interpretation of results, manuscript writing and editing, approved the final version. PVL: design, data collection, data analysis, interpretation of results, manuscript editing, approved the final version. JF: assistance with data analysis, interpretation of results, manuscript editing, approved the final version. MDL: interpretation of results, manuscript editing, approved the final version. PSdS: funding, conception, manuscript editing, approved the final version. SF: funding, conception, interpretation of results, manuscript writing and editing, approved the final version.

Funding The study was supported by a Crohn's \& Colitis Foundation of America (CCFA), Senior CCFA Research Award Number 112544.

Competing interests None declared.

Ethics approval The study was approved by the Danish Data Protection Agency (j.nr. 2014-41-3466). According to Danish law, no ethical approval of register-based studies is necessary.

Provenance and peer review Not commissioned; externally peer reviewed.

\section{REFERENCES}

1 Bernstein CN, Wajda A, Svenson LW, et al. The epidemiology of inflammatory bowel disease in Canada: a population-based study. Am I Gastroenterol 2006;101:1559-68.

2 Molodecky NA, Soon IS, Rabi DM, et al. Increasing incidence and prevalence of the inflammatory bowel diseases with time, based on systematic review. Gastroenterology 2012;142:46-54.e42; quiz e30.

3 Sjöberg D, Holmström T, Larsson M, et al. Incidence and natural history of ulcerative colitis in the Uppsala Region of Sweden 2005-2009—results from the IBD Cohort of the Uppsala Region (ICURE). J Crohns Colitis 2013;7:e351-7.

4 Shivananda S, Lennard-Jones J, Logan $R$, et al. Incidence of inflammatory bowel disease across Europe: is there a difference between north and south? Results of the European Collaborative Study on Inflammatory Bowel Disease (EC-IBD). Gut 1996;39:690-7

5 Norgard BM, Nielsen J, Fonager $\mathrm{K}$, et al. The incidence of ulcerative colitis (1995-2011) and Crohn's disease (1995-2012) —based on nationwide Danish registry data. J Crohns Colitis 2014;8:1274-80.

6 Rachootin P, Olsen J. Prevalence and socioeconomic correlates of subfecundity and spontaneous abortion in Denmark. Int J Epidemiol 1982;11:245-9.

7 Schmidt L. Infertility and assisted reproduction in Denmark. Epidemiology and psychosocial consequences. Dan Med Bull 2006;53:390-417.

8 Diamond MP, Freeman ML. Clinical implications of postsurgical adhesions. Hum Reprod Update 2001;7:567-76.

9 Johnson $\mathrm{P}$, Richard C, Ravid A, et al. Female infertility after ileal pouch-anal anastomosis for ulcerative colitis. Dis Colon Rectum 2004;47:1119-26.

10 Olsen $\mathrm{KO}$, Joelsson $\mathrm{M}$, Laurberg $\mathrm{S}$, et al. Fertility after ileal pouch-anal anastomosis in women with ulcerative colitis. Br J Surg 1999;86:493-5.

11 Ording Olsen $\mathrm{K}$, Juul $\mathrm{S}$, Berndtsson I, et al. Ulcerative colitis: female fecundity before diagnosis, during disease and after surgery compared with a population sample. Gastroenterology 2002;122:15-19.

12 Waljee A, Waljee J, Morris AM, et al. Threefold increased risk of infertility: a meta-analysis of infertility after ileal pouch anal anastomosis in ulcerative colitis. Gut 2006:55:1575-80.

13 Ban L, Tata LJ, Humes DJ, et al. Decreased fertility rates in 9639 women diagnosed with inflammatory bowel disease: a United Kingdom population-based cohort study. Aliment Pharmacol Ther 2015;42:855-66.

14 Wikland M, Jansson I, Asztely M, et al. Gynaecological problems related to anatomical changes after conventional proctocolectomy and ileostomy. Int $J$ Colorectal Dis 1990;5:49-52. 
15 Senates E, Colak Y, Erdem ED, et al. Serum anti-Mullerian hormone levels are lower in reproductive-age women with Crohn's disease compared to healthy control women. J Crohns Colitis 2013;7:e29-34.

16 Freour T, Miossec C, Bach-Ngohou K, et al. Ovarian reserve in young women of reproductive age with Crohn's disease. Inflamm Bowel Dis 2012;18:1515-22.

17 Pabby V, Oza SS, Dodge LE, et al. In vitro fertilization is successful in women with ulcerative colitis and ileal pouch anal anastomosis. Am J Gastroenterol 2015;110:792-7.

18 Oza SS, Pabby V, Dodge LE, et al. In vitro fertilization in women with inflammatory bowel disease is as successful as in women from the general infertility population. Clin Gastroenterol Hepatol 2015;13:1641-6.e3.

19 Pedersen CB, Gotzsche $\mathrm{H}$, Moller JO, et al. The Danish civil registration system. A cohort of eight million persons. Dan Med Bull 2006;53:441-9.

20 Andersen AN, Westergaard HB, Olsen J. The Danish in vitro fertilisation (IVF) register. Dan Med Bull 1999;46:357-60.

21 Westergaard HB, Johansen AM, Erb K, et al. Danish National IVF Registry 1994 and 1995. Treatment, pregnancy outcome and complications during pregnancy. Acta Obstet Gynecol Scand 2000;79:384-9.

22 Andersen TF, Madsen M, Jorgensen J, et al. The Danish National Hospital Register. A valuable source of data for modern health sciences. Dan Med Bull 1999;46:263-8.

23 Lynge E, Sandegaard JL, Rebolj M. The Danish national patient register. Scand J Public Health 2011;39(7 Suppl):30-3.

24 Petersen GL, Schmidt L, Pinborg A, et al. The influence of female and male body mass index on live births after assisted reproductive technology treatment: a nationwide register-based cohort study. Fertil Steril 2013;99:1654-62.

25 Fedder J, Loft A, Parner ET, et al. Neonatal outcome and congenital malformations in children born after ICSI with testicular or epididymal sperm: a controlled national cohort study. Hum Reprod 2013;28:230-40.

26 Stern JE, Brown MB, Wantman E, et al. Live birth rates and birth outcomes by diagnosis using linked cycles from the SART CORS database. J Assist Reprod Genet 2013;30:1445-50.

27 Knudsen LB, Olsen J. The Danish medical birth registry. Dan Med Bull 1998;45:320-3.

28 Kristensen J, Langhoff Roos J, Skovgaard LT, et al. Validation of the Danish birth registration. J Clin Epidemiol 1996;49:893-7.
29 Charlson ME, Pompei P, Ales KL, et al. A new method of classifying prognostic comorbidity in longitudinal studies: development and validation. J Chronic Dis 1987;40:373-83.

30 Winger $\mathrm{EE}$, Reed JL, Ashoush S, et al. Treatment with adalimumab (Humira) and intravenous immunoglobulin improves pregnancy rates in women undergoing IVF. Am J Reprod Immunol 2009;61:113-20.

31 Winger EE, Reed JL, Ashoush $\mathrm{S}$, et al. Degree of TNF- $\alpha / \mathrm{IL}-10$ cytokine elevation correlates with IVF success rates in women undergoing treatment with adalimumab (Humira) and IVIG. Am J Reprod Immunol 2011;65:610-18.

32 Winger EE, Reed JL, Ashoush $\mathrm{S}$, et al. Die-off ratio correlates with increased TNF- $\alpha$ : IL-10 ratio and decreased IVF success rates correctable with Humira. Am J Reprod Immunol 2012;68:428-37.

33 Mahadevan U, Sandborn WJ, Li DK, et al. Pregnancy outcomes in women with inflammatory bowel disease: a large community-based study from Northern California. Gastroenterology 2007;133:1106-12.

34 O'Toole A, Nwanne 0, Tomlinson T. Inflammatory bowel disease increases risk of adverse pregnancy outcomes: a meta-analysis. Dig Dis Sci 2015;60: 2750-61.

35 Kallen $C B$, Arici $A$. Immune testing in fertility practice: truth or deception? Curr Opin Obstet Gynecol. 2003;15:225.

36 Spor A, Koren 0, Ley R. Unraveling the effects of the environment and host genotype on the gut microbiome. Nat Rev Microbiol 2011;9:279-90.

37 Koren O, Goodrich JK, Cullender TC, et al. Host remodeling of the gut microbiome and metabolic changes during pregnancy. Cell 2012;150:470-80.

38 Konstantinov SR, van der Woude CJ, Peppelenbosch MP. Do pregnancy related changes in the microbiome stimulate innate immunity? Trends Mol Med 2013;19:454-9.

39 Jost $\mathrm{T}$, Lacroix $\mathrm{C}$, Braegger $\mathrm{C}$, et al. Stability of the maternal gut microbiota during late pregnancy and early lactation. Curr Microbiol 2014;68:419-27.

40 Mahadevan U, Martin CF, Sandler RS, et al. PIANO: a 1000 patient prospective registry of pregnancy outcomes in women with IBD exposed to immunomodulators and biologic therapy. Gastroenterology 2012;142:S-149.

41 Fonager K, Sorensen HT, Rasmussen SN, et al. Assessment of the diagnoses of Crohn's disease and ulcerative colitis in a Danish hospital information system. Scand J Gastroenterol 1996:31:154-9. 